\title{
Improved discrimination among similar agricultural plots using red-and-green-based pseudo-colour imaging
}

\author{
Ryoichi Doi \\ Faculty of Social-Human Environmentology, Daito Bunka University, 1-9-1 Takashimadaira, Itabashi-ku, Tokyo 175-8571, Japan
}

Received October 6, 2015; accepted March 23, 2016

\begin{abstract}
A b s t r a c t. The effects of a pseudo-colour imaging method were investigated by discriminating among similar agricultural plots in remote sensing images acquired using the Airborne Visible/ Infrared Imaging Spectrometer (Indiana, USA) and the Landsat 7 satellite (Fergana, Uzbekistan), and that provided by GoogleEarth (Toyama, Japan). From each dataset, red (R)-green (G)-R-Gblue yellow (RGrgbyB), and $\mathrm{RGrgby}^{-1} \mathrm{~B}$ pseudo-colour images were prepared. From each, cyan, magenta, yellow, key black, $L^{*}$, $a^{*}$, and $b^{*}$ derivative grayscale images were generated. In the Airborne Visible/Infrared Imaging Spectrometer image, pixels were selected for corn no tillage (29 pixels), corn minimum tillage (27), and soybean (34) plots. Likewise, in the Landsat 7 image, pixels representing corn (73 pixels), cotton (110), and wheat (112) plots were selected, and in the GoogleEarth image, those representing soybean (118 pixels) and rice (151) were selected. When the 14 derivative grayscale images were used together with an RGB yellow grayscale image, the overall classification accuracy improved from 74 to 94\% (Airborne Visible/Infrared Imaging Spectrometer), 64 to $83 \%$ (Landsat), or 77 to $90 \%$ (GoogleEarth). As an indicator of discriminatory power, the kappa significance improved $10^{18}$-fold (Airborne Visible/Infrared Imaging Spectrometer) or greater. The derivative grayscale images were found to increase the dimensionality and quantity of data. Herein, the details of the increases in dimensionality and quantity are further analysed and discussed.

K e y w o r d s: agricultural fields, colours of plant body, correlations and dimensionality, feasible multivariate imaging, multivariate analysis
\end{abstract}

\section{INTRODUCTION}

The precise remote sensing of agricultural fields is a goal of those involved in observation thereof. As processes of observation, observers perform acquisition, processing, and analysis of data such as remotely acquired images.
The process of acquisition has been significantly enhanced by increases in the spatial (Poli and Toutin, 2012) and spectral (Atzberger, 2013) resolution of sensors. The former and latter improvements were realized by high-resolution sensors like the one carried by the WorldView satellite and by multispectral sensors that have narrow bandwidths (such as every $5 \mathrm{~nm}$ ), respectively. In addition to the development of sensors, a significant increase in the number of alternatives for sensor carriers, such as unmanned aerial vehicles, was another major achievement that has resolved limitations in data acquisition (Matese et al., 2015). Methods of data analysis have also been advancing and have enhanced the effective use of acquired data. The supervised and unsupervised computation of pixels from acquired image data is the most widely applied tool for extracting the information carried by acquired data (Vibhute and Gawali, 2013). In addition to conventional pixel-based classification, object-based classification was introduced in the 1990s (Schotten et al., 1993). In addition to these major tools, there are other tools for the analysis of remote sensing data. For example, the normalized difference vegetation index and its variations indicate the activity and health of plants (Petorelli, 2013).

Compared to the above, the advancements in the processing of acquired remote sensing data before analysis seem limited. To date, one of the most intensively examined processing techniques has been the decorrelation of information carried by a multispectral dataset (Soha et al., 1976). For example, when a red (R)-green (G)-blue (B) true colour image is converted to an intensity-saturation-hue colour image, even when changes in the intensity 
of red, green, and blue are correlated, negligible correlations may be found among changes in intensity, saturation, and/ or hue. Thus, the amount of information may be increased by stretching the dark-bright distribution of the grayscale image for intensity, saturation, and/or hue (Gillespie et al., 1986). Image fusion is another technique that enhances the utilization of data from agricultural fields. Image fusion generates a single image from multiple images (Sahu and Parsai, 2012). The generated image is more informative and complete than any of the original images for a specific objective, such as identifying fruit disease problems (Dubey and Jalai, 2015).

As a tool for the processing of remote sensing images, a pseudo-colour imaging method was recently suggested as a new alternative for the precise observation of agricultural fields (Doi, 2014). The pseudo-colour imaging method generates pseudo-RGB colour images derived from grayscale images for the intensity of red and green. The pseudoRGB colour images are converted to cyan (C)-magenta (M)-yellow (Y)-key black (K), and International Commission on Illumination $L^{*} a^{*} b^{*}$ colour images. From the CMYK and $L^{*} a^{*} b^{*}$ colour images, grayscale images for cyan and the other colour components are prepared. These derivative grayscale images were suggested to enrich information on the grayscale intensity of pixels (Doi, 2014). The evidence of enrichment is shown as the non-linearity (Gillespie et al., 1986) between changes in the pixel grayscale intensity of some derivative grayscale images and those in the original grayscale images for red or green (Doi, 2014). This enrichment could result in a greater amount of information than that carried by the original images for multispectral bands. The power to discriminate among different objects is the most important requirement for the successful observation of agricultural fields (Zar, 1999). Thus, the enrichment of information was expected to enhance multispectral crop/field observation by increasing the power to discriminate among different objects.

The pseudo-imaging method has aspects in common with decorrelation and image fusion. As in decorrelation (Gillespie et al., 1986; Soha et al., 1976), the pseudo-colour imaging method extends the dimensionality of data by producing independent patterns of changes in the intensity of pixels in derivative grayscale images. Like image fusion, the pseudo-colour imaging method combines the original grayscale images for red and green, and generates a grayscale image for RGB yellow, which becomes a component of a pseudo-colour image. At the same time, the pseudo colour imaging method differs from decorrelation in that it generates pseudo-colour images that further generate derivative grayscale images. The pseudo-colour imaging method also differs from image fusion in that the latter is often used to improve the spatial resolution of data consisting of low-resolution images by relying on an image with greater spatial resolution (Liu et al., 2000). In contrast, the pseudo-colour imaging method is used to increase the quantity and dimensionality of information. A unique step in the pseudo-colour imaging method is replacing the grayscale image for blue in the original RGB colour image with a grayscale image for RGB yellow prepared from those for red and green. Image fusion provides a single image that contains the information needed by the user. In contrast, the pseudo-colour imaging method generates pseudo-colour RGB images, from which 14 derivative grayscale images are generated to enrich the information provided by the original dataset (such as multispectral images).

This study examined the increase in the discriminatory power of multispectral imaging using the pseudo-colour imaging method. The pseudo-colour imaging method was applied to the discrimination of difficult-to-discriminate agricultural plots in three remote sensing datasets. The first was a 1992 dataset from Tippecanoe County, Indiana, United States, acquired using the Airborne Visible/Infrared Imaging Spectrometer (AVIRIS). Corn plots under two management regimes and soybean plots were also discriminated between and among. The second dataset included cotton, corn, and wheat plots from the Fergana Valley of Uzbekistan. The Fergana dataset consisted of seven images acquired in 2011 using Landsat 7. The third was an RGB true colour image of experimental rice and soybean plots in Toyama city, Japan. The Toyama dataset was captured in 2012 and is publically available through GoogleEarth. The contribution of derivative grayscale images generated by the pseudo-colour imaging method was evaluated in terms of classification accuracy and an increase in the dimensionality of dataset. For grayscale images that significantly contributed to the discrimination, the detailed mechanism is further discussed.

\section{METHODS}

Imagery data acquired by AVIRIS in the agricultural area of Montmorenci in Tippecanoe County, Indiana, United States (Fig. 1), were downloaded from the MultiSpec website of Purdue University (https://engineering.purdue. edu/ biehl/MultiSpec/). The AVIRIS data were acquired on 12 June 1992. The digital data were recorded as 220 hyperspectral grayscale images of $145 \times 145$ pixels square. The images were saved as TIFF data. Each pixel represented a $20 \times 20 \mathrm{~m}$ square (Tadjudin and Landgrebe, 1996). Among the 220 images, six were selected to prepare a dataset like those acquired by commercially available multispectral sensors (Turner et al., 2014). The selected bands were blue (wavelength $479 \mathrm{~nm}$, band 9), green $(557 \mathrm{~nm}$, band 17), red (657 $\mathrm{nm}$, band 27), near-infrared $(822 \mathrm{~nm}$, band 46), mid-infrared $1(1.65 \mu \mathrm{m}$, band 133$)$, and midinfrared $2(2.20 \mu \mathrm{m}$, band 190$)$.

Satellite imagery data acquired by the Landsat 7 satellite in the Fergana Valley of Uzbekistan were downloaded from the US Global Survey site (http://www.usgs.gov/). The Landsat 7 data were acquired on 11 July 2011. The 

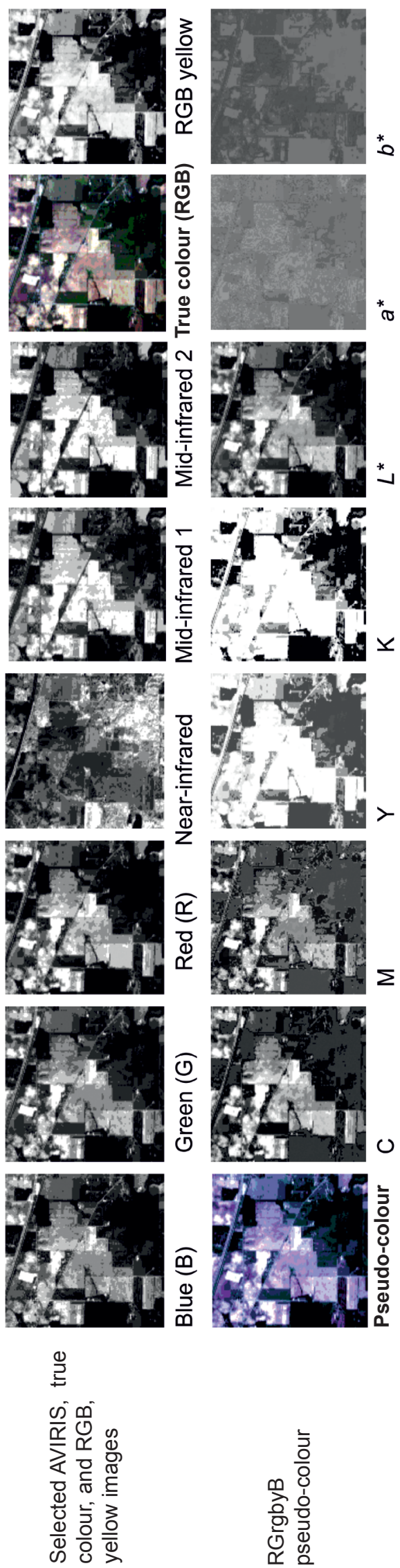

*
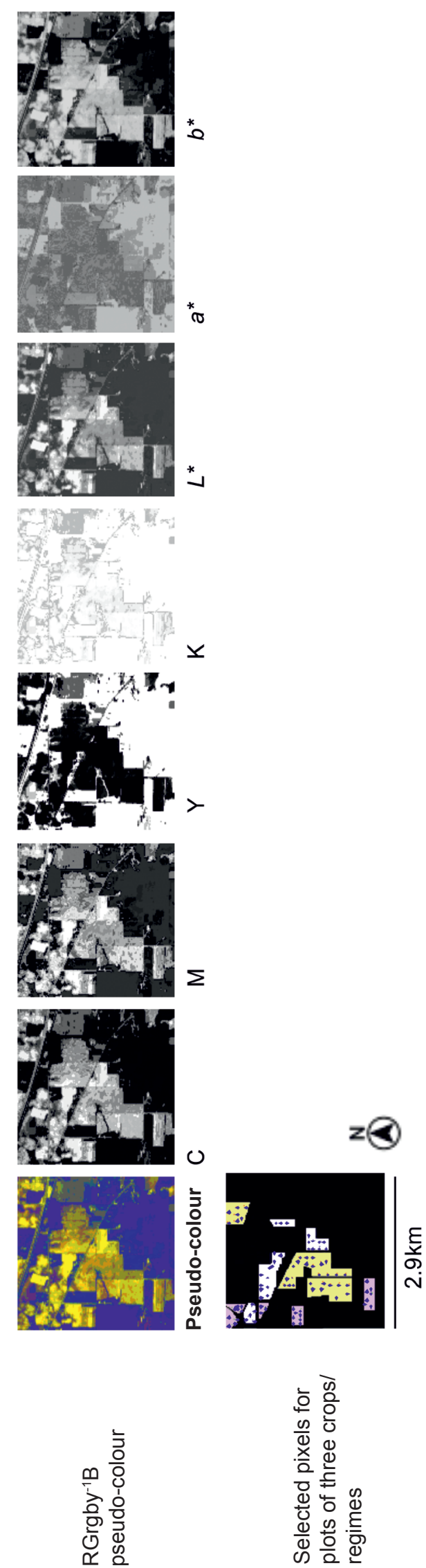

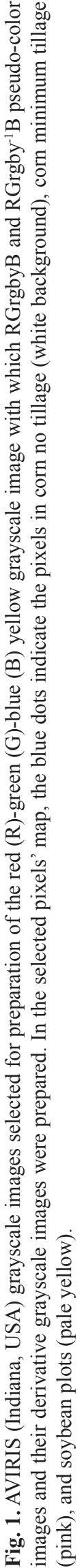


data were recorded as TIFF grayscale images for bands of blue (450-515 nm, band 1), green (525-605 nm, band 2), red (630-890 $\mathrm{nm}$, band 3), near-infrared (750-900 $\mathrm{nm}$, band 4), mid-infrared $1(1.55-1.75 \mu \mathrm{m}$, band 5), thermal infrared (10.4-12.5 $\mu \mathrm{m}$, band 6), mid-infrared 2 (2.09-2.35 $\mu \mathrm{m}$, band 7), and panchromatic data $(0.52-0.90 \mu \mathrm{m}$, band 8$)$. In the image, a rectangular-shaped area was selected. The represented area was $11.25 \times 20 \mathrm{~km}$. Each pixel represented a $30 \times 30 \mathrm{~m}$ square. The ground-truth information was obtained from the Central Asian Water Initiative website (http://www.cawa-project.net/). The grayscale images for bands 1 to 7 were used.

The GoogleEarth image of the experimental plots in Toyama, Japan, was captured on 27 July 2012. When copying the remote sensing image from the GoogleEarth window, the 'copy image' function was used. When the image was copied from the GoogleEarth window, the data regarding the values of the red (R)-green (G)-blue (B) colour intensity were retained. The image data was then pasted into a new file window in Adobe Photoshop 7.0. In the Adobe Photoshop window, a single pixel represented an area of $82 \times 82 \mathrm{~cm}$. The image was saved as an RGB TIFF file. The copied and saved image represented a $601 \times 1087 \mathrm{~m}$ rectangle. The ground-truth information was provided as an official document of the Agricultural Research Institute of Toyama prefecture, Japan.

For each dataset, with Adobe Photoshop 7.0 computer software, grayscale images for red and green were used to prepare R-G-RGB yellow (RGrgbyB) and R-G-inverted RGB yellow (RGrgby $\left.{ }^{-1} \mathrm{~B}\right)$ pseudo-colour images (Doi, 2014). The images were saved as TIFF files in the RGB colour mode. In this study, the Adobe RGB colour space was chosen as one of the RGB colour spaces. An RGB yellow grayscale image was prepared by merging the $\mathrm{R}$ and $\mathrm{G}$ grayscale images (Doi, 2012). Each of the RGrgbyB and $\mathrm{RGrgby}^{-1} \mathrm{~B}$ pseudo-colour TIFF images was converted to cyan (C)-magenta (M)-yellow (Y)-key black (K) and International Commission on Illumination $L^{*} a * b *$ images. CMYK images were generated using the International Colour Consortium profile of US Web Coated (SWOP) v2 for digital output such as colour printing. Next, grayscale images that showed the intensity values of C, M, Y, K, and $L^{*}$ and the values of $a^{*}$ and $b^{*}$ were prepared (Fig. 1, Doi et $a l ., 2014)$. Hereafter, the $\mathrm{C}, \mathrm{M}, \mathrm{Y}, \mathrm{K}, L^{*}, a^{*}$, and $b^{*}$ images are referred to as derivative grayscale images. Thus, 15 grayscale images were added to the six Indiana AVIRIS or the seven Fergana Landsat 7 images, including the RGB yellow image. The original RGB image of Toyama was converted to CMYK and $L^{*} a * b *$ true colour images. Then, grayscale images for $\mathrm{C}, \mathrm{M}, \mathrm{Y}, \mathrm{K}, L^{*}, a^{*}$, and $b^{*}$ were prepared in addition to a grayscale image for RGB yellow and the other 14 derivative grayscale images from RGrgbyB and $\mathrm{RGrgby}^{-1} \mathrm{~B}$ pseudo-colour images, as in the case of the AVIRIS (Indiana) and Landsat 7 (Fergana) datasets. In this manner, the GoogleEarth (Toyama) dataset eventually consisted of 25 grayscale images.
These 21 (AVIRIS, Indiana), 22 (Landsat 7, Fergana), or 25 (GoogleEarth, Toyama) grayscale images were used in the experiment to compare the power to discriminate pixels that represent different crops/management regimes in the remote sensing image. Among the pixels in the image, the author selected pixels representing different crops/management regimes. In the Indiana image, pixels representing corn no tillage, corn minimum tillage, and soybean were selected (Fig. 1). The numbers of pixels were 29, 27, and 34 for corn no tillage, corn minimum tillage, and soybean, respectively (Manian and Jimenez, 2007). Likewise, in the Fergana image, 73, 110, and 112 pixels were selected for corn, cotton, and wheat, respectively. In the Toyama image, 118 and 151 pixels representing soybean and rice, respectively, were selected.

The grayscale intensity profiles of the pixels were analysed using the pixel analysis software, MultiSpec 3.3 (Purdue Research Foundation), to examine the improvement of classification accuracy by the inclusion of the RGB yellow and 14 derivative grayscale images in addition to the original AVIRIS, Landsat 7, or GoogleEarth images. Using the statistical software SPSS 10.0.1 (SPSS Inc.), principal component analysis was also performed to analyse the data structure on the grayscale intensity profiles of the selected pixels for the 21 (AVIRIS), 22 (Landsat), or 25 (GoogleEarth) grayscale images. Using the SPSS software, the kappa statistic and its significance were also determined.

\section{RESULTS AND DISCUSSION}

With the six AVIRIS images from Indiana, discriminating among the three groups of pixels was relatively more difficult (Table 1) than that with all 21 images. Bandos et al. (2009) have previously demonstrated the difficulty in discriminating among these pixels using linearity-based computation. Using the six images, the overall accuracy in the classification of all 90 pixels was $74 \%$. This unsuccessful discrimination seems to be attributable to the negligible crop canopies at the time point of the image data acquisition on 12 June 1992. In the region, soybean is planted from May to mid-June (Boyer et al., 2015) whereas corn is conventionally planted in May (Schenk and Barber, 1980). When the image was captured, the plants were so small that the differences in leaf colour contributed to the discrimination only slightly (Fig. 1). Due to the undeveloped canopies, the discrimination had to rely largely on soil profiles. Even in a single plot, the profiles of pixels representing soil can vary more than those of crop leaves (Todd and Hoffer, 1998). This soil-caused intra-plot variation in each of the plots is believed to have made the discrimination difficult (Fig. 1). The discrimination was more successful with the derivative and the RGB yellow grayscale images, resulting in $94 \%$ overall accuracy. The value of the kappa statistic improved from 0.612 (substantial agreement, 
T a b l e 1. Classification of the selected pixels representing different crops/management regimes in the AVIRIS (Indiana) image (Fig. 1) based on the grayscale intensity profiles of the pixels. Combinations of the six AVIRIS grayscale images and all 21 grayscale images are compared in terms of classification accuracy

\begin{tabular}{|c|c|c|c|c|c|c|c|c|c|}
\hline \multirow{3}{*}{$\begin{array}{l}\text { Grayscale } \\
\text { images } \\
\text { used }\end{array}$} & \multicolumn{2}{|c|}{ True crop/management } & \multicolumn{3}{|c|}{ Classified crops/management } & \multirow{3}{*}{ Total } & \multirow{3}{*}{$\begin{array}{c}\text { Accuracy } \\
(\%)\end{array}$} & \multirow{3}{*}{$\begin{array}{c}\text { Overall } \\
\text { accuracy* } \\
(\%)\end{array}$} & \multirow{3}{*}{$\begin{array}{l}\text { Kappa } \\
\text { statistic } \\
\text { (kappa } \\
\text { significance } \\
\text { as p value) }\end{array}$} \\
\hline & \multirow{2}{*}{ Crop } & \multirow{2}{*}{$\begin{array}{l}\text { Mana- } \\
\text { gement } \\
\text { regime }\end{array}$} & \multicolumn{2}{|c|}{ Corn } & \multirow[b]{2}{*}{ Soybean } & & & & \\
\hline & & & No tillage & $\begin{array}{c}\text { Minimum } \\
\text { tillage }\end{array}$ & & & & & \\
\hline \multirow{3}{*}{$\begin{array}{l}\text { Original six } \\
\text { grayscale } \\
\text { images** }\end{array}$} & \multirow[b]{2}{*}{ Corn } & No tillage & 18 & 6 & 5 & 29 & 62 & \multirow[t]{2}{*}{74} & 0.612 \\
\hline & & Minimum tillage & 1 & 20 & 6 & 27 & 74 & & $\left(1.49 \times 10^{-16}\right)$ \\
\hline & Soybean & & 2 & 3 & 29 & 34 & 85 & & \\
\hline \multirow{3}{*}{$\begin{array}{l}\text { All } 21 \\
\text { (original six, } \\
\text { RGB yellow, } \\
\text { and } 14 \\
\text { derivatives) }\end{array}$} & \multirow{2}{*}{ Corn } & No tillage & 29 & 0 & 0 & 29 & 100 & \multirow[t]{3}{*}{94} & \multirow{3}{*}{$\begin{array}{l}0.917 \\
\left(6.68 \times 10^{-35}\right.\end{array}$} \\
\hline & & Minimum tillage & 1 & 26 & 0 & 27 & 96 & & \\
\hline & Soybean & & 3 & 1 & 30 & 34 & 88 & & \\
\hline
\end{tabular}

*Overall accuracy - sum of properly classified pixels (white characters in the black cells) for the three crops/management among the total number of selected pixels (90) (Fig. 1, bottom left); ** grayscale intensity representing reflectance for blue, green, red, nearinfrared, and mid-infrared 1 and 2.

T a b l e 2. Best combinations of grayscale images for classification of the selected pixels in the AVIRIS (Indiana) image (Fig. 1) based on differences among crops and crop management regimes

\begin{tabular}{|c|c|c|c|c|}
\hline \multirow{2}{*}{$\begin{array}{l}\text { Number of } \\
\text { grayscale } \\
\text { images } \\
\text { combined }\end{array}$} & \multirow[b]{2}{*}{ Best combinations of grayscale images } & \multicolumn{3}{|c|}{ Inter-crop/management distance* } \\
\hline & & $\begin{array}{l}\text { Corn no tillage- } \\
\text { corn minimum } \\
\text { tillage }\end{array}$ & $\begin{array}{l}\text { Corn no } \\
\text { tillage-soybean }\end{array}$ & $\begin{array}{l}\text { Corn minimum } \\
\text { tillage-soybean }\end{array}$ \\
\hline & RGrgbyB K & 0.85 & 1.33 & 0.22 \\
\hline \multirow[t]{3}{*}{1} & RGrgbyB Y & 0.17 & 0.53 & 0.17 \\
\hline & Green & 0.03 & 0.10 & 0.03 \\
\hline & RGrgbyB Y, RGrgbyB K & 1.93 & 1.35 & 0.90 \\
\hline \multirow[t]{3}{*}{2} & RGrgbyB K, RGrgby-1B Y & 1.53 & 1.38 & 0.49 \\
\hline & RGrgbyB K, RGrgby-1B b* & 1.17 & 1.39 & 0.31 \\
\hline & Near-infrared, RGrgbyB Y, RGrgbyB K & 1.95 & 1.48 & 0.96 \\
\hline \multirow[t]{3}{*}{3} & Mid-infrared 2, RGrgbyB Y, RGrgbyB K & 2.01 & 1.35 & 0.95 \\
\hline & RGrgbyB Y, RGrgbyB K, RGrgby-1B K & 2.14 & 1.68 & 0.94 \\
\hline & Near-infrared, mid-infrared 2, RGrgbyB Y, RGrgbyB K & 2.10 & 1.52 & 1.24 \\
\hline \multirow[t]{2}{*}{4} & Near-infrared, RGrgbyB M, RGrgbyB Y, RGrgbyB K & 2.00 & 1.73 & 1.15 \\
\hline & Near-infrared, mid-infrared 1, RGrgbyB Y, RGrgbyB K & 2.07 & 1.50 & 1.12 \\
\hline
\end{tabular}

*The greater the distance, the more powerful the discrimination power for the combination of grayscale images. 
T a b l e 3. Structure of data on grayscale intensity profiles of the 90 selected pixels in the AVIRIS (Indiana) image (Fig. 1, bottom left) revealed as eigenvectors and other statistics of principal components

\begin{tabular}{|c|c|c|c|c|c|}
\hline \multirow{3}{*}{$\begin{array}{l}\text { Direct source of } \\
\text { grayscale image }\end{array}$} & \multirow{3}{*}{$\begin{array}{l}\text { Grayscale image in which } \\
\text { grayscale intensitywas read } \\
\text { to profile the } 90 \text { selected } \\
\text { pixels }\end{array}$} & \multicolumn{4}{|c|}{ Principal component* (variation explained and eigenvalue) } \\
\hline & & 1 & 2 & 3 & 4 \\
\hline & & $(70 \%, 14.8)$ & $(12 \%, 2.46)$ & $(7 \%, 1.49)$ & $(3 \%, 0.73)$ \\
\hline \multirow{6}{*}{ Original AVIRIS data } & Blue & $0.85 * *$ & -0.21 & 0.18 & 0.20 \\
\hline & Green $(G)$ & 0.98 & -0.17 & -0.04 & 0.09 \\
\hline & Red (R) & 0.93 & 0.04 & 0.33 & 0.15 \\
\hline & Near-infrared & 0.85 & -0.16 & -0.02 & -0.07 \\
\hline & Mid-infrared 1 & 0.69 & 0.10 & 0.25 & -0.48 \\
\hline & Mid-infrared 2 & 0.74 & 0.11 & 0.38 & -0.17 \\
\hline $\begin{array}{l}\mathrm{R} \text { and } \mathrm{G} \text { grayscale } \\
\text { images of the original } \\
\text { AVIRIS data }\end{array}$ & RGB yellow & 0.96 & 0.23 & 0.04 & -0.06 \\
\hline \multirow{7}{*}{$\begin{array}{l}\text { RGrgbyB pseudo- } \\
\text { colour image }\end{array}$} & Cyan & 0.97 & 0.03 & 0.20 & 0.07 \\
\hline & Magenta & 0.98 & -0.15 & -0.14 & 0.00 \\
\hline & Yellow & 0.60 & 0.77 & -0.21 & 0.06 \\
\hline & Key black & 0.42 & 0.78 & -0.19 & 0.22 \\
\hline & $L^{*}$ & 0.99 & -0.08 & 0.04 & 0.09 \\
\hline & $a^{*}$ & -0.65 & 0.51 & 0.55 & 0.00 \\
\hline & $b^{*}$ & 0.81 & -0.49 & 0.06 & 0.25 \\
\hline \multirow{7}{*}{$\begin{array}{l}\text { RGrgby }^{-1} \mathrm{~B} \text { pseudo- } \\
\text { colour image }\end{array}$} & Cyan & 0.88 & 0.20 & 0.35 & 0.18 \\
\hline & Magenta & 0.95 & -0.06 & -0.27 & -0.03 \\
\hline & Yellow & -0.70 & -0.55 & 0.26 & 0.24 \\
\hline & Key black & 0.66 & -0.35 & 0.15 & -0.39 \\
\hline & $L^{*}$ & 0.98 & -0.13 & 0.05 & 0.09 \\
\hline & $a^{*}$ & -0.75 & 0.16 & 0.63 & 0.07 \\
\hline & $b^{*}$ & 0.97 & 0.21 & -0.02 & -0.04 \\
\hline
\end{tabular}

*The most significant four principal components are listed, **eigenvector.

Viera and Garrett, 2005) to 0.917 (almost perfect agreement). The $p$ value also showed a marked improvement of $2.2 \times 10^{18}$-fold. In the improvement of the discrimination, some derivative grayscale images were found to have contributed significantly (Table 2). For example, RGrgbyB $\mathrm{K}$ and RGrgbyB Y grayscale images were the largest contributors. The contribution in discriminating among the pixel groups was obviously due to an increase in the dimensionality of the dataset on the grayscale intensity profiles of the 90 pixels (Table 3 ).

Among all 21 variables, RGrgbyB K and RGrgbyB Y were the greatest contributors to the second principal component, and the original six AVIRIS variables contributed heavily to the first principal component and only slightly 
T a b l e 4. Classification of the selected pixels representing different crops in the Landsat 7 (Fergana) image based on the grayscale intensity profiles of the pixels. Combinations of the seven Landsat 7 grayscale images and all 22 grayscale images are compared in terms of classification accuracy

\begin{tabular}{|c|c|c|c|c|c|c|c|c|}
\hline \multirow{2}{*}{ Grayscale images used } & \multirow{2}{*}{$\begin{array}{l}\text { True } \\
\text { crop }\end{array}$} & \multicolumn{3}{|c|}{ Classified crops } & \multirow{2}{*}{ Total } & \multirow{2}{*}{$\begin{array}{l}\text { Accuracy } \\
(\%)\end{array}$} & \multirow{2}{*}{$\begin{array}{l}\text { Overall } \\
\text { accuracy* } \\
\quad(\%)\end{array}$} & \multirow{2}{*}{$\begin{array}{c}\text { Kappa statistic } \\
\text { (kappa } \\
\text { significance } \\
\text { as p value) }\end{array}$} \\
\hline & & Corn & Cotton & Wheat & & & & \\
\hline \multirow{3}{*}{$\begin{array}{l}\text { Original seven grayscale } \\
\text { images } * *\end{array}$} & Corn & 33 & 26 & 14 & 73 & 47 & 64 & 0.450 \\
\hline & Cotton & 28 & 61 & 21 & 110 & 64 & & $\left(1.65 \times 10^{-27}\right)$ \\
\hline & Wheat & 9 & 8 & 95 & 112 & 73 & & \\
\hline \multirow{3}{*}{$\begin{array}{l}\text { All } 22 \text { (original seven, red- } \\
\text { green-blue yellow, and } 14 \\
\text { derivative images) }\end{array}$} & Corn & 61 & 4 & 8 & 73 & 82 & 83 & 0.736 \\
\hline & Cotton & 8 & 85 & 17 & 110 & 87 & & $\left(1.80 \times 10^{-70}\right)$ \\
\hline & Wheat & 5 & 9 & 98 & 112 & 80 & & \\
\hline
\end{tabular}

*Overall accuracy - sum of properly classified pixels (white characters in the black cells) for the three crops / total number of selected pixels (295); **grayscale intensity representing reflectance for blue, green, red, near-infrared, thermal, and mid-infrared 1 and 2.

T a b l e 5. Classification of the selected pixels representing different crops in the GoogleEarth (Toyama) image based on the grayscale intensity profiles of the pixels. Three combinations of grayscale images are compared in terms of classification accuracy

\begin{tabular}{|c|c|c|c|c|c|c|c|}
\hline \multirow{2}{*}{ Grayscale images used } & \multirow{2}{*}{$\begin{array}{l}\text { True } \\
\text { crop }\end{array}$} & \multicolumn{2}{|c|}{ Classified crops } & \multirow{2}{*}{ Total } & \multirow{2}{*}{$\begin{array}{l}\text { Accuracy } \\
(\%)\end{array}$} & \multirow{2}{*}{$\begin{array}{l}\text { Overall } \\
\text { accuracy }{ }^{1} \\
(\%)\end{array}$} & \multirow{2}{*}{$\begin{array}{c}\text { Kappa statistic } \\
\text { (kappa } \\
\text { significance } \\
\text { as p value) }\end{array}$} \\
\hline & & Soybean & Rice & & & & \\
\hline \multirow{2}{*}{$\begin{array}{l}\text { Grayscale images for red }(R) \text {, } \\
\text { green }(G) \text {, and blue }(B)\end{array}$} & Soybean & 88 & 30 & 118 & 69 & 74 & 0.483 \\
\hline & Rice & 39 & 112 & 151 & 79 & & $\left(1.91 \times 10^{-15}\right)$ \\
\hline \multirow{2}{*}{$\begin{array}{l}\text { Grayscale images for RGB, } \\
\text { CMYK, and } L^{*} a^{*} b^{*},{ }^{2}\end{array}$} & Soybean & 87 & 31 & 118 & 74 & 77 & 0.532 \\
\hline & Rice & 31 & 120 & 151 & 80 & & $\left(2.66 \times 10^{-18}\right)$ \\
\hline \multirow{2}{*}{$\begin{array}{l}\text { Grayscale images for all } 25 \\
\text { colour components }\end{array}$} & Soybean & 107 & 11 & 118 & 87 & 90 & 0.797 \\
\hline & Rice & 16 & 135 & 151 & 93 & & $\left(4.11 \times 10^{-39}\right)$ \\
\hline
\end{tabular}

${ }^{1}$ Overall accuracy - sum of properly classified pixels (white characters in the black cells) for the crops / total number of selected pixels (269); ${ }^{2}$ grayscale images for the intensity of R, G, B, cyan (C), magenta (M), yellow (Y), key black (K), and $L^{*}$ and the values of $a^{*}$ and $b^{*}$ as components of the true color GoogleEarth image.

to the second. This contrast indicates an increase in the dimensionality provided by the RGrgbyB K and RGrgbyB $\mathrm{Y}$ grayscale images that improved the discriminatory power by providing additional minor but significant measures (de Sena et al., 2000; Doi and Sakurai, 2004). The minor principal components usefully indicate differences among similar pixels that are hard to discriminate based on the six AVIRIS variables that have strong linear correlations to one another and with the first principal component. Thus, the data structure is simple, based on the six variables only
(Table 3). Table 3 and the visual appearance of the images in Fig. 1 indicate the differences between the RGrgbyB and $\mathrm{RGrgby}^{-1} \mathrm{~B}$ pseudo-colour images. These differences suggest further opportunities to find differences among similar pixels by utilizing the pseudo-colour models.

In the discrimination among plots of the three crops in the Landsat 7 image of the Fergana Valley, the derivative grayscale images improved the discrimination (Table 4). The overall classification accuracy increased from 64 to $83 \%$. The kappa statistic and its significance also markedly 


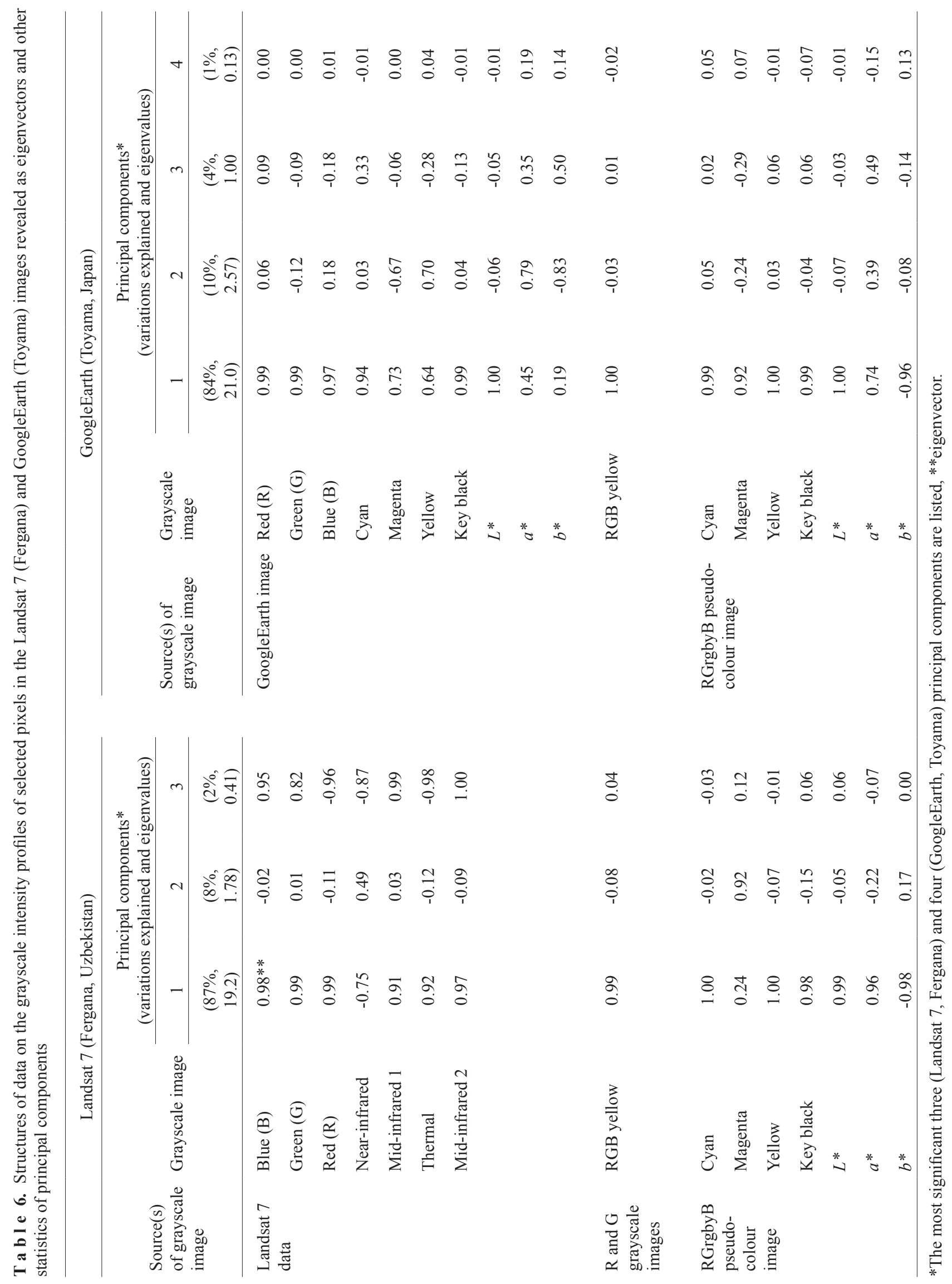




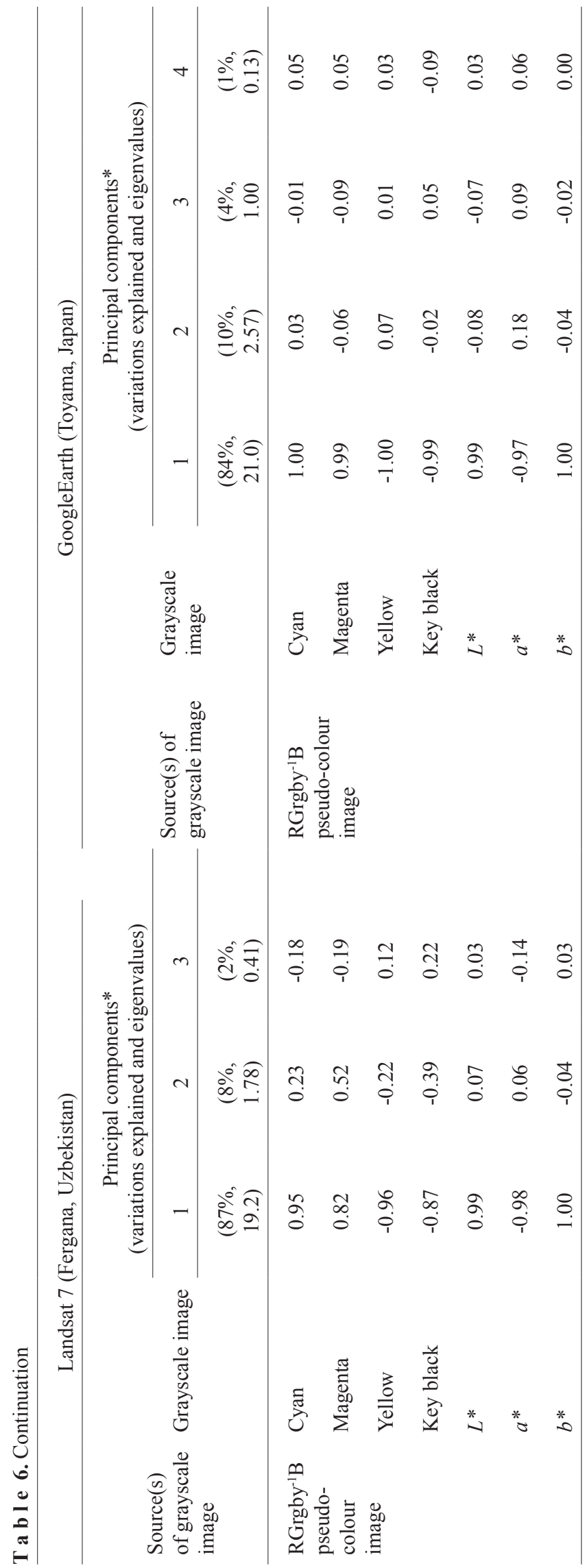

improved from 0.450 to 0.736 and from $1.65 \times 10^{-27}$ to $1.80 \times 10^{-70}$, respectively. When the original seven Landsat 7 images were used, the corn and cotton plots were especially difficult to discriminate. This difficulty was thought to be caused by the vigorously growing green leaves of the crops at the time point of image acquisition on 11 July 2011 (Asia Development Bank, 2013). This difficulty was largely circumvented by using the derivative grayscale images. Analysing the GoogleEarth dataset on the Toyama experimental plots also resulted in a similar and significant improvement (Table 5). When the only grayscale images for the intensity of red, green, and blue were used, the overall classification accuracy was $74 \%$; this was slightly improved to $77 \%$ by using 10 grayscale images for RGB, CMYK, and $L^{*} a * b *$ as components of the true colour image. With the 10 grayscale images, the changes in the values of the kappa statistic and its significance were also slight, compared to the AVIRIS (Indiana) and Landsat 7 (Fergana) datasets (Tables 1, 4). However, when 14 derivative grayscale images were used together with an RGB yellow grayscale image, the classification accuracy significantly improved. The involvement of all 25 grayscale images resulted in an overall accuracy percentage of $90 \%$ (Table 5). The kappa statistic also improved from 0.483 ( $R, G$, and B grayscale images only) to 0.797 , and the significance improved $4.64 \times 10^{23}$-fold. The discrimination between soybean and rice plots was expected to be difficult because of the weather condition, with obviously visible interference by clouds. Another expected disadvantage was that the number of original grayscale images was only three. Despite these limitations, the derivative grayscale images generated by the pseudo-colour imaging method aided in the discrimination significantly.

Structures of the Landsat 7 (Fergana) and GoogleEarth (Toyama) datasets are shown in Table 6. The datasets are similar in that, for the first principal components, the absolute values of eigenvectors for the derivative grayscale images are comparably large, compared to those for the original grayscale images of the Landsat 7 and GoogleEarth data. This indicates that the derivative grayscale images played important roles in the discrimination, and that the information provided by them was not secondary and minor but was major and powerful. The significant contribution of the derivative grayscale images was evidenced by the great increase in the significance of the kappa statistic for the Landsat 7 and the GoogleEarth datasets (Tables 4, 5). In terms of changes in the kappa statistic and its significance, the derivative grayscale images generated from the Landsat 7 and the GoogleEarth datasets improved the discrimination more significantly than in the case of the AVIRIS dataset on the Indiana site (Tables 1, 3). The grayscale images that contributed most significantly to the discrimination among the plots of the Fergana Valley or the 
T a b l e 7. Best combinations of grayscale images for classification of the selected pixels in the Landsat 7 (Fergana, Uzbekistan) and GoogleEarth (Toyama, Japan) images based on spectral differences among crops

\begin{tabular}{|c|c|c|}
\hline Dataset & $\begin{array}{l}\text { Number of grayscale } \\
\text { images combined }\end{array}$ & Best combinations of grayscale images \\
\hline \multirow[t]{12}{*}{ Landsat 7 (Fergana, Uzbekistan) } & 1 & RGrgbyB K \\
\hline & & Red \\
\hline & & $\operatorname{RGrgby}^{-1} \mathrm{~B} b^{*}$ \\
\hline & 2 & $\mathrm{RGrgby}^{-1} \mathrm{~B} \mathrm{M}, \mathrm{RGrgby}^{-1} \mathrm{~B} L^{*}$ \\
\hline & & Mid-infrared 2, RGrgbyB $b^{*}$ \\
\hline & & Green, RGrgby ${ }^{-1} \mathrm{~B} \mathrm{M}$ \\
\hline & 3 & Green, Thermal, RGrgbyB $L^{*}$ \\
\hline & & Thermal, RGrgby $^{-1} \mathrm{~B} \mathrm{M}, \mathrm{RGrgby}^{-1} \mathrm{~B} L^{*}$ \\
\hline & & RGrgbyB $b^{*}, \mathrm{RGrgby}^{-1} \mathrm{~B} \mathrm{M}, \mathrm{RGrgby}^{-1} \mathrm{~B} L^{*}$ \\
\hline & 4 & Green, RGrgbyB C, RGrgbyB M, RGrgby ${ }^{-1} \mathrm{~B} b^{*}$ \\
\hline & & RGrgbyB $b^{*}, \operatorname{RGrgbyB}^{\mathrm{M}}, \mathrm{RGrgby}^{-1} \mathrm{~B} \mathrm{~K}, \mathrm{RGrgby}^{-1} \mathrm{~B} b^{*}$ \\
\hline & & Green, Thermal, RGrgbyB $L^{*}, \mathrm{RGrgby}^{-1} \mathrm{~B} \mathrm{M}$ \\
\hline \multirow[t]{12}{*}{ GoogleEarth (Toyama, Japan) } & 1 & $a^{*}$ \\
\hline & & $\mathrm{RGrgby}^{-1} \mathrm{~B} \mathrm{~K}$ \\
\hline & & RGrgbyB C \\
\hline & 2 & Blue, RGrgby ${ }^{-1} \mathrm{~B}$ Y \\
\hline & & Key black, RGrgby ${ }^{-1} \mathrm{~B}$ Y \\
\hline & & Blue, RGrgby $^{-1} \mathrm{~B} b^{*}$ \\
\hline & 3 & Key black, RGrgbyB K, RGrgby ${ }^{-1} \mathrm{~B}$ Y \\
\hline & & Blue, RGrgbyB K, RGrgby ${ }^{-1}$ B Y \\
\hline & & Blue, RGrgby $^{-1} \mathrm{~B} \mathrm{M}, \mathrm{RGrgby}^{-1} \mathrm{~B} b^{*}$ \\
\hline & 4 & Blue, RGB yellow, RGrgby $^{-1} \mathrm{~B}$ C, RGrgby $^{-1} \mathrm{~B}$ Y \\
\hline & & Key black, RGB yellow, RGrgby ${ }^{-1} \mathrm{~B}$ C, RGrgby $^{-1} \mathrm{~B}$ Y \\
\hline & & Blue, RGB yellow, RGrgby ${ }^{-1} \mathrm{~B}$ Y, RGrgby $^{-1} \mathrm{~B}$ K \\
\hline
\end{tabular}

Toyama experimental fields are listed in Table 7. Again, several derivative grayscale images were found to be the best to discriminate among/between the plots.

To further investigate the effects of derivative grayscale images on the increase in the dimensionality and quantity of information, RGrgbyB $\mathrm{K}$ and RGrgbyB Y grayscale images of the Indiana site were prepared in combination with grayscale images for standard RGB yellow (Fig. 2). Standard grayscale intensity gradients indicating the intensity values of 0 (complete black), 50, 100, 150, 200 , and 255 (complete white) for redness and greenness were merged to prepare 36 RGB yellowness standard grids.
Correlations between the grayscale intensity of RGB yellowness and changes in the grayscale intensity among the 36 grids for RGrgbyB K, RGrgbyB Y, redness, and greenness were compared in terms of information overlapping in a diagram (Fig. 3). As the intensity of greenness and redness has a strong correlation with RGB yellowness (Doi, 2012, Fig. 3), linear relationships are seen among them (Figs 2 and 3). In contrast, the changes in the grayscale intensity of pixels in the RGrgbyB K and RGrgbyB Y images in Fig. 2 were quite different from those in the grayscale intensity of the $\mathrm{R}$ and $\mathrm{G}$ images in Fig. 2. It is evident that the patterns of the changes in the grayscale intensity of pixels in the 


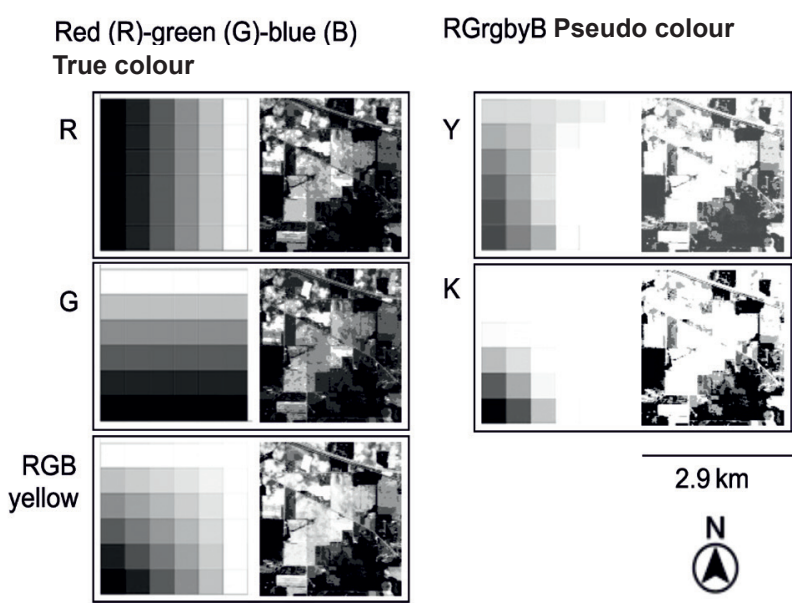

Fig. 2. Standard color intensity gradients (left) and corresponding image of the AVIRIS (Indiana) image (right) shown as grayscale images for selected color components of the true color RGB and the RGrgbyB pseudo-colour models.

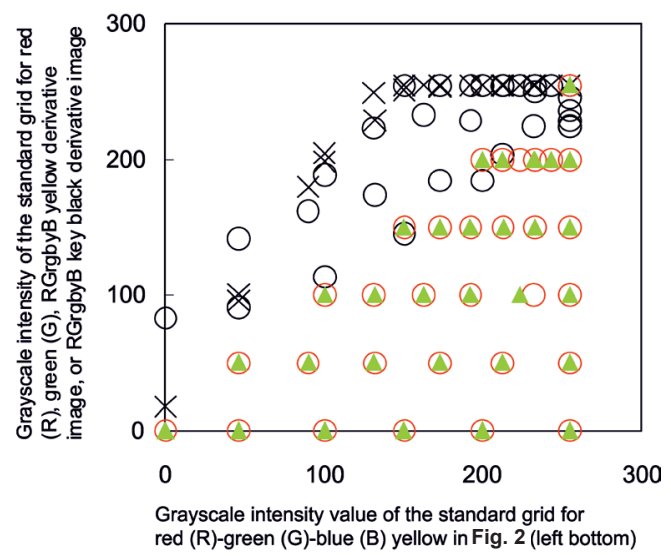

Fig. 3. Relationships between changes in the grayscale intensity of the standard grids of red (R)-green (G)-blue (B) yellow (Fig. 2, bottom left) and those for $\mathrm{R}(\mathrm{O}), \mathrm{G}(\boldsymbol{\Delta})$, and derivative grayscale images for yellow $(\times)$ and key black $(O)$ (Fig. 2, right) as components of the RGrgbyB pseudo-colour image shown in Fig. 1.

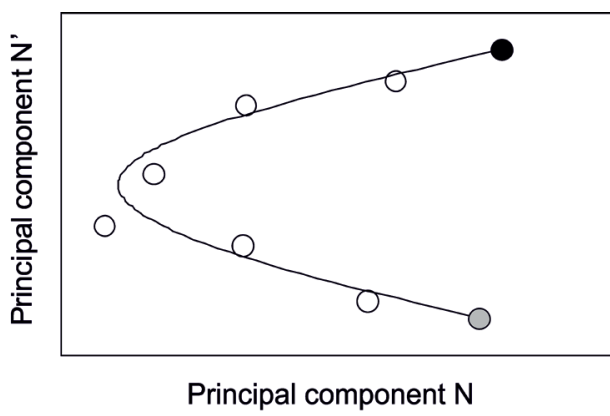

Fig. 4. Non-linearity among significant principal components helps discrimination among difficult-to-discriminate objects by reflectance observation. In this schematic diagram, the solid (O) and gray (O) symbols indicate samples are hard to discriminate based on their principal component $\mathrm{N}$ scores, but they are clearly distinguished based on their principal component N' scores.
RGrgbyB K and RGrgbyB Y images compensated for the difficulty in detecting changes in grayscale intensity in the original $\mathrm{R}$ and $\mathrm{G}$ images (Fig. 2). RGB yellow is a colour shown as a sum of the intensity of redness and greenness. Because redness and greenness are known to describe a large portion of changes in the colour of a plant body (Parsons et al., 2009), the range of change in RGB yellow (Fig. 2, bottom left) should express the colours of most plant bodies. Therefore, the RGB colour model should have worked as long as changes in the intensity of redness, greenness, and blueness had linear correlations with important changes in the colour of plant organs, soils, and other objects in agricultural fields. However, as indicated by the above results (Tables 1 to 7), the linearity cannot always be expected. Rather, in this study, the discrimination was improved by the unique changes in the grayscale intensity of the derivative grayscale images (Fig. 3). The advantage of the derivative grayscale images is the independence of the changes in the pixel intensity from those in the original AVIRIS, Landsat 7, and GoogleEarth images (Doi, 2013, Hayes and Sader, 2001). Even when grayscale changes in the original grayscale images are linearly correlated, as in the case of the AVIRIS Indiana data (Table 3), the derivative grayscale images that have non-linear relationships with the original changes offer more chances to detect other patterns of spectral changes. Hence, the non-linearity was advantageous to the detection of changes in the images to be analysed, as demonstrated in Figs 2 and 3 (Fig. 4).

The derivative grayscale images were demonstrated to enrich the information carried by the AVIRIS, Landsat 7, and GoogleEarth datasets. The applicability of the pseudocolour imaging method was previously reported through the observation of a rice canopy using a commercially available anti-theft surveillance camera (Doi et al., 2014). This example and the cases of the AVIRIS, Landsat 7, and GoogleEarth datasets indicate the general applicability of the pseudo-colour imaging method to improve discrimination among difficult-to-discriminate objects in agricultural fields. Computation hardware and the architecture for the analysis of remote sensing data are currently developing rapidly and with a variety of computation algorithms (Bhojne et al., 2013). Furthermore, various remote sensing sensors and instruments to carry these sensors are becoming more commercially available. In addition to improvements in spatial and spectral resolution in remote sensing, significant improvement in temporal resolution is underway as various real-time remote sensing tools are entering the market (Ehsani et al., 2012; Verhoeven, 2009). The pseudo-colour imaging method is highly feasible because a computer and free software such as GIMP are all that is needed to enable users to take advantage of the pseudocolour imaging method. Therefore, under the aforementioned technological circumstances, remote sensing users may easily combine various remote sensing tools and the pseudo-colour imaging method. 


\section{CONCLUSIONS}

1. The pseudo-colour imaging method increased the discriminatory power of multispectral imaging and enhanced the discrimination among pixels representing similar and hard-to-discriminate agricultural plots.

2. The improvement was attributed to some of the 14 derivative grayscale images derived from pseudo-colour images.

3. The increases in the dimensionality and quantity of information were important intermediate results that provided the improvement in the discrimination.

4. The fundamental strength of the pseudo-colour imaging method is attributed to: a) non-linearity among changes in the grayscale intensity of pixels in some derivative grayscale images; thus b) the patterns of changes in the derivative grayscale images do not correlate with those in the original grayscale images of the AVIRIS, Landsat 7, and GoogleEarth datasets.

5. The general applicability of the pseudo-colour imaging method was demonstrated by examining the method for the discrimination of pixels representing different agricultural plots in the AVIRIS, Landsat 7, and GoogleEarth images.

\section{REFERENCES}

Asian Development Bank, 2013. Republic of Uzbekistan: Amu Bukhara irrigation system rehabilitation (feasibility study). Technical Assistance Consultant's Report, Manila.

Atzberger C., 2013. Advances in remote sensing of agriculture: Context description, existing operational monitoring systems and major information needs. Remote Sens., 5, 949-981.

Bandos T.V., Bruzzone L., and Camps-Valls G., 2009. Classification of hyperspectral images with regularized linear discriminant analysis. IEEE Trans. Geosci. Remote Sens., 47, 862-873.

Bhojne M., Chakravarti A., Pallav A., and Sivakumar V., 2013. High performance computing for satellite image processing and analyzing - A review. Int. J. Comput. Appl. Tech Res., 2, 424-430.

Boyer C.N., Stefanini M., Larson J.A., Smith S.A., Mengistu A., and Bellaloui N., 2015. Profitability and risk analysis of soybean planting date by maturity group. Agron. J., 107, 2253-2262.

de Sena M.M., Poppi R.J., Frighetto R.T.S., and Valarini P.J., 2000. Evaluation of the use of chemometric methods in soil analysis. Quim. Nova, 23, 547-556.

Doi R., 2012. Simple luminosity normalization of greenness, yellowness and redness/greenness for comparison of leaf spectral profiles in multi-temporally acquired remote sensing images. J. Biosci., 37, 723-730.

Doi R., 2013. Discriminating crop and other canopies by overlapping binary image layers. Opt. Eng., 52, Article number 020502.

Doi R., 2014. Red-and-green-based pseudo-RGB color models for the comparison of digital images acquired under different brightness levels. J. Modern Opt., 61, 1373-1380.
Doi R., Arif C., Setiawan B.I., and Mizoguchi M., 2014. Pixel color clustering of multi-temporally acquired digital photographs of a rice canopy by luminosity-normalization and pseudo-red-green-blue color imaging. Sci. World J., Article number 450374.

Doi R. and Sakurai K., 2004. Principal components derived from soil physico-chemical data explained a land degradation gradient, and suggested the applicability of new indexes for estimation of soil productivity in the Sakaerat Environmental Research Station, Thailand. Int. J. Sust. Dev. World Ecol., 11, 298-311.

Dubey S.R. and Jalal A.S., 2015. Application of image processing in fruit and vegetable analysis: A review. J. Intell. Syst., $24,405-424$.

Ehsani R., Sankaran S., Maja J.M., and Neto J. C., 2012. Affordable multi-rotor remote sensing platform for applications in precision horticulture. Proc. 11th Int. Conf. Precision Agriculture, July 15-18, Indianapolis, USA.

Gillespie A.R., Kahle A.B., and Walker R.E., 1986. Color enhancement of highly correlated images. I. Decorrelation and HSI contrast stretches. Remote Sens. Environ., 20, 209-235.

Hayes D. and Sader S., 2001. Comparison of change-detection techniques for monitoring tropical forest clearing and vegetation regrowth in a time series. Photogramm. Eng. Remote Sensing, 67, 1067-1075.

Liu Q-J., Tateishi R., Kondoh A., and Takeuchi N., 2000. Vegetation types classification of a temperate biosphere reserve in China by multisensor satellite imagery. J. Japan Society Photogrammetry Remote Sensing, 39, 23-34.

Manian V. and Jimenez L.O., 2007. Land cover and benthic habitat classification using texture features from hyperspectral and multispectral images. J. Electronic Imaging, 16, Article number 023011 .

Matese A., Toscano P., Di Gennaro S.F., Genesio L., Vaccari F.P., Primicerio J., Belli C., Zaldei A., Bianconi R., and Gioli B., 2015. Intercomparison of uav, aircraft and satellite remote sensing platforms for precision viticulture. Remote Sensing, 7, 2971-2990.

Parsons N.R., Edmondson R., and Song Y., 2009. Image analysis and statistical modelling for measurement and quality assessment of ornamental horticulture crops in glasshouses. Biosyst. Eng., 104, 161-168.

Pettorelli N., 2013. The normalized difference vegetation index. Oxford University Press, UK.

Poli D. and Toutin T., 2012. Review of developments in geometric modelling for high resolution satellite pushbroom sensors. Photogrammetric Record, 27, 58-73.

Sahu D.K. and Parsai M., 2012. Different image fusion techniques-a critical review. Int. J. Modern Eng. Res., 2, 4298-4301.

Schenk M. and Barber S., 1980. Potassium and phosphorus uptake by corn genotypes grown in the field as influenced by root characteristics. Plant Soil, 54, 65-76.

Soha J.M., Gillespie A.R., Abrams M.J., and Madura D.P., 1976. Computer techniques for geological applications. Proc. Caltech/JPL Conference on Image Processing Technology, Data Sources and Software for Commercial and Scientific Applications, November 3-5, Caltech, Pasadena, CA, USA. 
Schotten C.G.J., Janssen L., and Nieuwenhuis G.J., 1993. Comparison of optical and microwave satellite data for land cover inventory purposes using digital field boundaries. EARSeL Advances in Remote Sensing, 2, 22-26.

Tadjudin S. and Landgrebe D., 1996. A decision tree classifier design for high-dimensional data with limited training samples. Proc. IEEE Geoscience and Remote Sensing Symposium, May 27-31, Lincoln, NE, USA. .

Todd S.W. and Hoffer R.M., 1998. Responses of spectral indices to variations in vegetation cover and soil background. Photogramm. Eng. Remote Sensing, 64, 915-922.

Turner D., Lucieer A., Malenovský Z., King D.H., and Robinson S.A., 2014. Spatial co-registration of ultra-high resolution visible, multispectral and thermal images acquired with a micro-UAV over Antarctic Moss Beds. Remote Sens., 6, 4003-4024.

Verhoeven G.J.J., 2009. Providing an archaeological bird's-eye view - an overall picture of ground-based means to execute low-altitude aerial photography (LAAP) in archaeology. Archaeological Prospection, 16, 233-249.

Vibhute A.D. and Gawali B.W., 2013. Analysis and modeling of agricultural land use using remote sensing and geographic information system: A review. Int. J. Eng. Res. Applications (IJERA), 3, 81-91.

Viera A.J. and Garrett J.M., 2005. Understanding interobserver agreement: the kappa statistic. Fam. Med., 37, 360-363.

Zar J.H., 1999. Biostatistical analysis, Pearson Education, India. 